\title{
Transforming Gas Giant Planets into Smaller Objects Through Tidal Disruption
}

\author{
Shang-Fei Liu ${ }^{1, *}$, James Guillochon ${ }^{2}$, \\ Douglas N. C. Lin ${ }^{1,2}$ and Enrico Ramirez-Ruiz ${ }^{2}$ \\ *email: liushangfei@pku.edu.cn \\ ${ }^{1}$ Dept. of Astronomy and Kavli Institute for Astronomy \& Astrophysics, \\ Peking University, Beijing 100871, China \\ ${ }^{2}$ Dept. of Astronomy \& Astrophysics, University of California, Santa Cruz, \\ CA 95064, U.S.A.
}

\begin{abstract}
Recent observations have revealed several Jupiter-mass planets with highly eccentric and / or misaligned orbits, which clearly suggests that dynamical processes operated in these systems. These dynamical processes may result in close encounters between Jupiter-like planets and their host stars. Using three-dimensional hydrodynamical simulations, we find that planets with cores are more likely to be retained by their host stars in contrast with previous studies which suggested that coreless planets are often ejected. We propose that after a long term evolution some gas giant planets could be transformed into super-Earths or Neptune-like planets, which is supported by our adiabatic evolution models. Finally, we analyze the orbits and structure of known planets and Kepler candidates and find that our model is capable of producing some of the shortest-period objects.
\end{abstract}

Keywords. Tidal disruption, hot Jupiters, super-Earths, planetary structure

\section{Introduction}

A widely adopted scenario of the origin of close-in planets is based on the assumption that all gas giant planets formed beyond the snow line a few AU from their host star (Pollack et al. 1996), with the progenitors of hot Jupiters undergoing substantial inward migration through planet-disk interaction (Lin et al. 1996). This mechanism naturally leads to the formation of resonant gas giants and coplanarity between the planets' orbits and their natal disks. However, measurements of the Rossiter-McLaughlin effect (Ohta et al. 2005) reveal that the orbits of a sub population of hot Jupiters appear to be misaligned with the spin of their host stars (Winn et al. 2010), which poses a challenge to the disk-migration scenario for the origin of hot Jupiters (Triaud et al. 2010).

In order to reconcile the theoretical predictions with the observations, some dynamical processes have been proposed, such as the Kozai mechanism (Kozai 1996), planet-planet scattering (Rasio \& Ford 1996) or secular chaos (Wu \& Lithwick 2011). The observed eccentricity distribution of extra-solar planets with periods longer than a week and masses larger than that of Saturn has a median value noticeably deviated from zero. Presumably they obtained this eccentricity through dynamical instability after the depletion of their natal disks (Lin \& Ida 1997), as the eccentricity damping would suppress such an instability if they were embedded in a gaseous disk environment.

Some of these processes can produce planets that lie on nearly parabolic orbits. As their eccentricity approaches unity, planets with a semimajor axis of a few AU undergo close encounters with their host stars. Hydrodynamical simulations have been carried out by Faber et al. (2005, hereafter FRW) and Guillochon et al. (2011, hereafter GRL) to study 

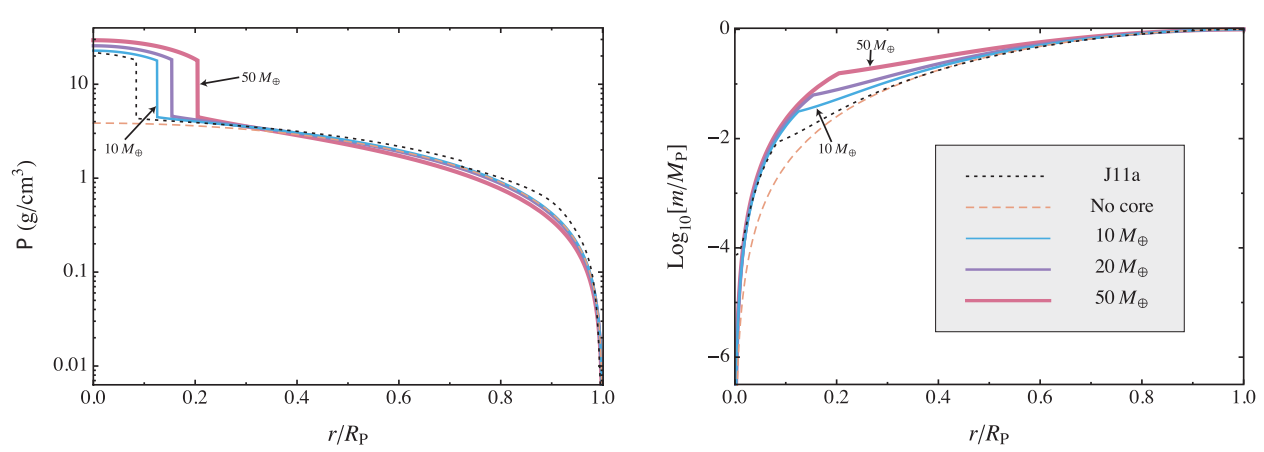

Figure 1. One-dimensional profiles of density (left) and enclosed mass (right) of a composite polytropic model $\left(n_{1}=0.5\right.$ and $\left.n_{2}=1\right)$ for a Jupiter-mass planet with no core, a $10 M_{\oplus}$ core, a $20 M_{\oplus}$ core, and a $50 M_{\oplus}$ core.

the survivability and orbital evolution of a Jupiter-mass planet disrupted by a Sun-like star. Our previous simulations of single nearly parabolic (with $e \simeq 1$ ) encounters show that within a sufficiently close range, planets are ejected due to mass and energy loss near periastron. For more distant periastron encounters, we showed that after successive encounters within a critical periastron separation no planet can avoid destruction. We re-examine the disruption and retention of gas giant planets by taking into account the presence of their dense cores. We show that presence of a core with mass as small as $10 M_{\oplus}$, the planet has a far greater chance of survival, even with a mass loss comparable to its total mass. We also consider the possibility that the tidal disruption mechanism may be an efficient way to transform a Jupiter-mass planet into a close-in super-Earth or Neptune-like object, which potentially may explain the existence of some of the inner edge of close-in planets.

\section{A Composite Polytrope Model for Gas Giant Planets with Cores}

For computational simplicity, we approximate core-envelope structure of gas giant planets by a composite polytrope model. We adopt the approach used in Rappaport et al. (1983) with the incorporation of different species to model the transition in the composition and EOS at the core-envelope interface in giant planets. To model the giant planets with cores, we choose the polytropic indices to be $n_{1}=0.5$ and $n_{2}=1$. Thus their continuity equations become

$$
\frac{\xi_{1 \mathrm{i}} \theta_{1 \mathrm{i}}^{n_{1}}}{\theta_{1 \mathrm{i}}^{\prime} \mu_{1}}=\frac{\xi_{2 \mathrm{i}} \theta_{2 \mathrm{i}}^{n_{2}}}{\theta_{2 \mathrm{i}}^{\prime} \mu_{2}},\left(\frac{n_{1}+1}{n_{2}+1}\right) \frac{\xi_{1 \mathrm{i}} \theta_{1 \mathrm{i}}^{\prime}}{\theta_{1 \mathrm{i}} \mu_{1}}=\frac{\xi_{2 \mathrm{i}} \theta_{2 \mathrm{i}}^{\prime}}{\theta_{2 \mathrm{i}} \mu_{2}},
$$

where $\mu_{1}$ and $\mu_{2}$ are the mean molecular weight in the core and the envelope.

In this work, we generate three composite polytrope models for a Jupiter-like planet with core masses of $10 M_{\oplus}, 20 M_{\oplus}$ and $50 M_{\oplus}$. Fig. 1 shows the density and mass distribution of these models (solid colored lines), where a constant $\mu_{1}=4 \mu_{2}$ has been assumed and $\xi_{1 \mathrm{i}}$ are chosen to be $1.571,1.799$ and 2.064 , respectively. The orange dashed line indicates the single-layered polytrope model, and the black dotted line shows a three-layer model for Jupiter taken from Nettelmann et al. (2008), which includes a 2.75 $M_{\oplus}$ core. Though the models presented here have more massive cores, our composite polytrope models generally fit the three-layer model very well, whereas the single-layered polytrope fails to represent the high density of the core. 


\section{Hydrodynamical Simulations of Tidal Disruption}

Our simulations are constructed based on the framework of FLASH (Fryxell et al. 2000), an adaptive-mesh, grid-based hydrodynamics code. The total volume of the simulation box is $10^{13} \times 10^{13} \times 10^{13} \mathrm{~cm}^{3}$. The initial conditions are identical to that of FRW and GRL to facilitate comparisons. The planet is assumed to have a mass $M_{\mathrm{P}}=M_{\mathrm{J}}$ and a radius $R_{\mathrm{P}}=R_{\mathrm{J}}$, where $M_{\mathrm{J}}$ and $R_{\mathrm{J}}$ are Jovian mass and radius, respectively. The planets are disrupted by a star with $M_{*}=10^{3} M_{\mathrm{J}} \simeq 0.95 M_{\odot}$. In the description of the relative strength of the tidal field exerted on a planet by the host star, it is useful to define a characteristic tidal radius as $r_{\mathrm{t}} \equiv\left(M_{*} / M_{\mathrm{P}}\right)^{1 / 3} R_{\mathrm{P}}$. Thus, the tidal radius of the planet is $r_{\mathrm{t}}=10 R_{\mathrm{J}} \simeq 0.995 R_{\odot}=0.00463 \mathrm{AU}$.

In total, we simulated 41 models with the three different core masses and the initial periastron distance $r_{\mathrm{p}}$ ranging from 1.15 to $2.5 r_{\mathrm{t}}$. A selection of simulations for $M_{\text {core }}=10 M_{\oplus}$ is illustrated in Fig. 2 .

In all our simulations, the planet is placed on a bound orbit with a negative orbital energy per unit mass $E_{\text {orb, } 0}$. We plot in the left panel of Fig. 3 the ratio of $E_{\text {orb }} / E_{\text {orb }, 0}$, where $E_{\text {orb }}$ is the energy per unit mass at the end of the simulation, approximately 50 dynamical timescales after pericenter. A planet's orbit is more (less) gravitationally bound to its host star if this ratio attains a positive value greater (lesser) than unity. A planet becomes unbound if this ratio attains a negative value. For comparison with previous simulations, we show the results obtained by FRW with open squares and those of single-layered polytropes obtained by

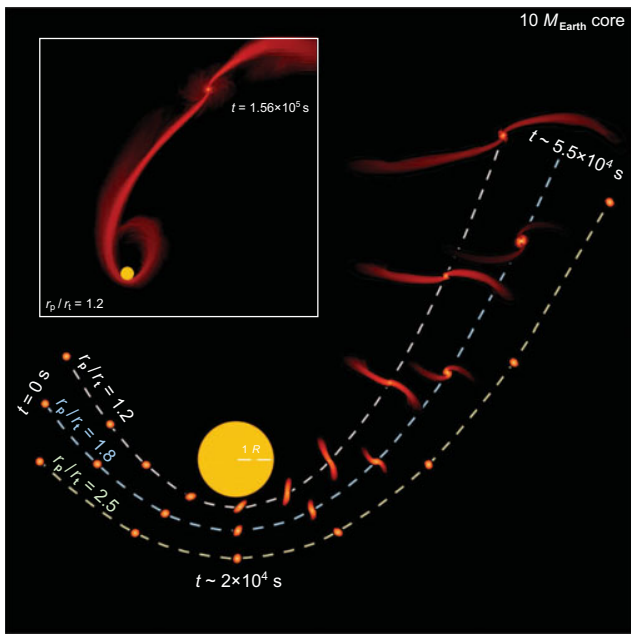

Figure 2. Snapshots from several simulations of the tidal disruption of a Jupiter mass planet with a $10 M_{\oplus}$ core at different periastron distances.

GRL with orange dashed lines in Fig. 3. The results of the new simulations with $10 M_{\oplus}$, $20 M_{\oplus}$ and $50 M_{\oplus}$ cores are shown as colored solid lines.
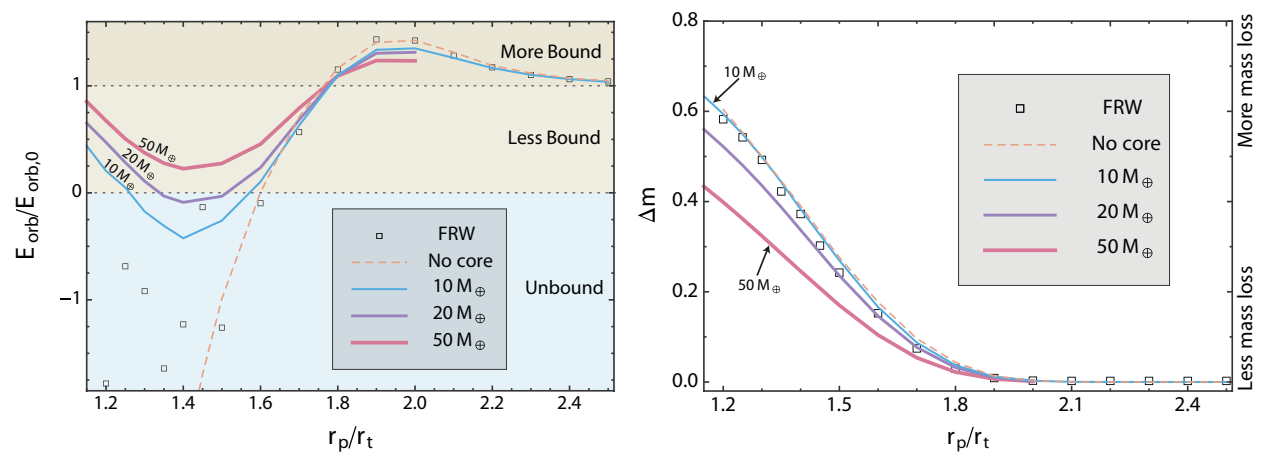

Figure 3. Final specific orbital energy $E_{\text {orb }}$ scaled to the initial specific orbital energy $E_{\text {orb }, 0}$ (left panel). Total mass fraction lost from Jupiter-like planets of varying core masses as a function of $r_{\mathrm{p}} / r_{\mathrm{t}}$. (right panel) 

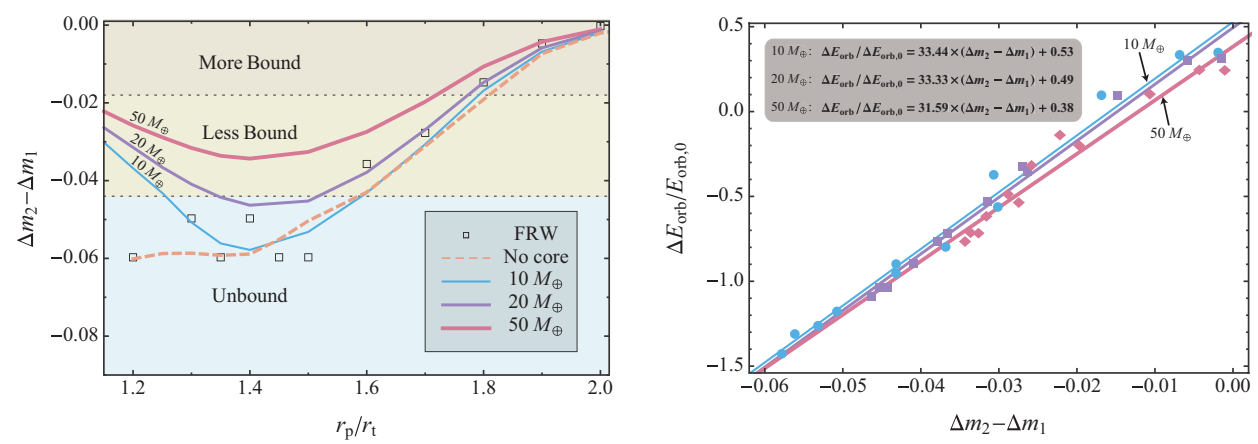

Figure 4. The left panel shows the difference between the two normalized mass loss $\Delta m_{2}-\Delta m_{1}$ as a function of periastron distance scaled by the tidal radius $r_{\mathrm{p}} / r_{\mathrm{t}}$. The correlation between the mass loss difference $\Delta m_{2}-\Delta m_{1}$ and changes in orbital energy scaled to the initial specific orbital energy $\Delta E_{\text {orb }} / E_{\text {orb }, 0}$ is shown in the right panel, where the orange triangles, blue points, purple squares and red diamonds are the simulation data of planets with no core, $10 M_{\oplus}, 20 M_{\oplus}$ and $50 M_{\oplus}$ cores respectively. The blue thin, purple medium, and red thick lines show the linear least squares fits to the simulations with cores.

An intriguing aspect of the work presented here is that if a dense core is present, a giant planet can remain bound to the star within certain limits of periastron separation, whereas previous simulations (e.g., FRW and GRL) suggested that planets without a core are always ejected or destroyed if any mass is lost during the initial inspiral. The presence of the core permits planets to plunge deeply into their parent star's tidal field and potentially survive as a close-in planet on a circular orbit.

The fraction of mass unbound from the planet $\Delta m$ in each run is plotted in the right panel of Fig. 3. The results show that for encounters with $r_{\mathrm{p}} \geqslant 2.0 r_{\mathrm{t}}$, tides raised by the star are too weak to shed any noticeable amount of mass from the planet. However, in the mass-shedding regime $\left(r_{\mathrm{p}}<2.0 r_{\mathrm{t}}\right)$, the discrepancies between different models are rather prominent. Planets with bigger cores lose significantly less amount of mass at the same periastron distance. We also calculate the asymmetry of the mass loss in two tidal streams and confirm that the change in specific orbital energy is linearly correlated with the asymmetric mass loss as shown in Fig. 4.

Planets with cores not only lose less mass, but also maintain
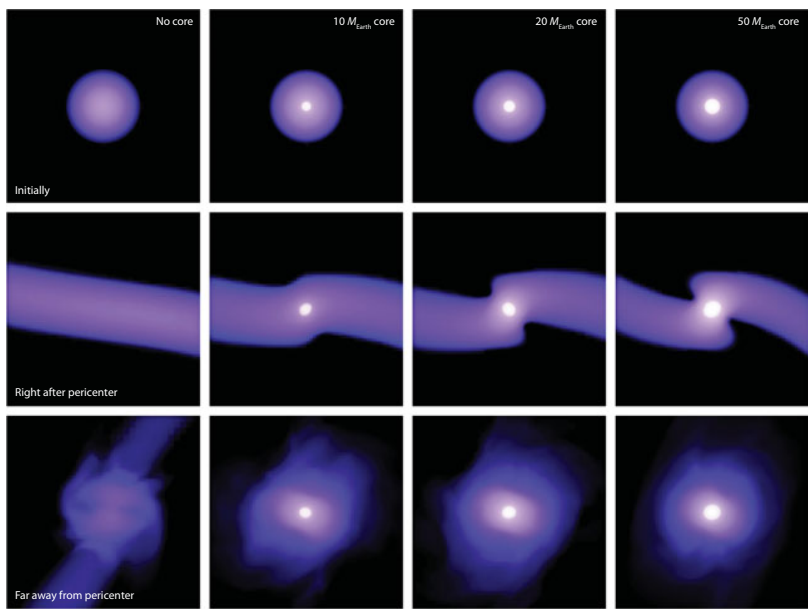

Figure 5. Snapshots from disruption simulations of Jupiter-mass giant planets with different core masses, modeled as a dual-layered composite polytropes. Four simulations are shown above (one per column), with the only difference between the simulations being the mass of the planet's core (as labeled). The planet comes within $1.2 r_{\mathrm{t}}$ of its parent star in each of the simulations. their internal structures more effectively than their coreless counterparts after the disruption has occurred (Fig. 5). This effect is important in determining the fate of tidal disrupted planets. 


\section{Discussion \& Summary}

We use the adiabatic response model which was outlined by Hjellming \& Webbink (1987) to investigate the long term evolution of mass-losing planets. A stiff EOS $\left(n_{1}=\right.$ 0.01 ) is required in order to capture the core's incompressible response to pressure deformations. In contrast to the single-layered $n=1$ polytrope, which has a constant radius $\left(R_{\mathrm{P}}\right.$ does not change with decreasing $\left.M_{\mathrm{P}}\right)$, composite polytropes with a small $n_{1}$ always contract as they lose mass $\left(R_{\mathrm{P}}\right.$ decreases significantly when $M_{\mathrm{P}}$ becomes small) as illustrated in Fig. 6.

The $n=1$ single-layered polytrope, which corresponds to the coreless gas giant planets, does not change its radius when losing mass adiabatically, resulting in a decrease of the average density. By contrast, the extremely incompressible cores of composite polytropes are weakly affected by the perturbation, imposing an almost constant inner boundary condition for the envelope, and resulting in an increase in density when the core's gravity dominates. This phenomenon helps to explain the different amounts of mass lost in the two cases. Each time the single-layered polytrope loses some mass, the specific gravitational self-binding energy decreases, leading to a more tidally-vulnerable structure. As a result, GRL found that coreless planets are always destroyed after several passages even if the initial periastron is fairly distant (the lower limit is $2.7 r_{\mathrm{t}}$ ). The composite polytropes, on the other hand, maintain a constant gravitational potential well in their centers, which continuously resists the stellar tidal force. Being invulnerable to tidal disruption themselves, the cores survive, retaining some fraction of the original envelope. Eventually when the disrupted remnant becomes dense enough or far away from the tidal radius to the parent star, it can reside around its parent star.

In order to search for some clues on the role of tidal disruption during their orbital circularization process, we show a sample of exoplanets with known planetary radii $R_{\mathrm{P}}$ and masses $M_{\mathrm{P}}$ and known stellar masses $M_{*}$ in the left panel of Fig. 7, where the distribution of the planet's mass as a function of its pericenter distance scaled by its tidal radius

$$
\frac{r_{\mathrm{p}}}{r_{\mathrm{t}}}=\frac{a(1-e)}{\left(M_{*} / M_{\mathrm{P}}\right)^{1 / 3} R_{\mathrm{P}}}
$$

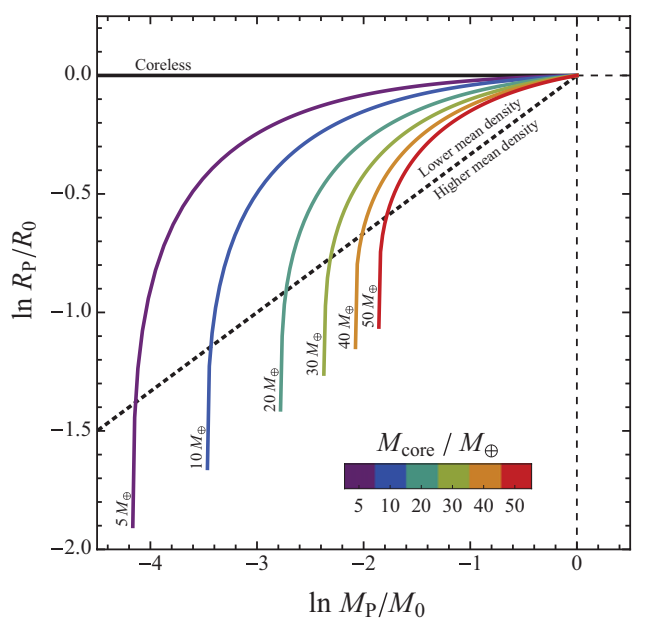

Figure 6. Adiabatic response curves for composite polytropes for varying degrees of mass loss. $M_{0}$ and $R_{0}$ denote the original mass and radius. A negative value indicates either a decrease in mass (x-axis) or a shrinkage of the planet's radius (y-axis). The top horizontal thick black line shows the evolution for a single-layered $n=1$ polytrope model.

is plotted. The color-coding of the filled dots denotes eccentricity $e$, and the open black circles represent planets with unknown eccentricity, where we have assumed $e=0$ for our subsequent calculations. The size of the symbols is representative of the planet's physical size (not drawn to scale). The tracks illustrate how tidally mass-losing planets with different core masses evolve.

Of the transiting super-Earths with known masses, only a few presently lie within a few tidal radii of their host stars. This may indicate that the conditions necessary to generate such planets via tidal disruption are uncommonly realized in nature. However, 

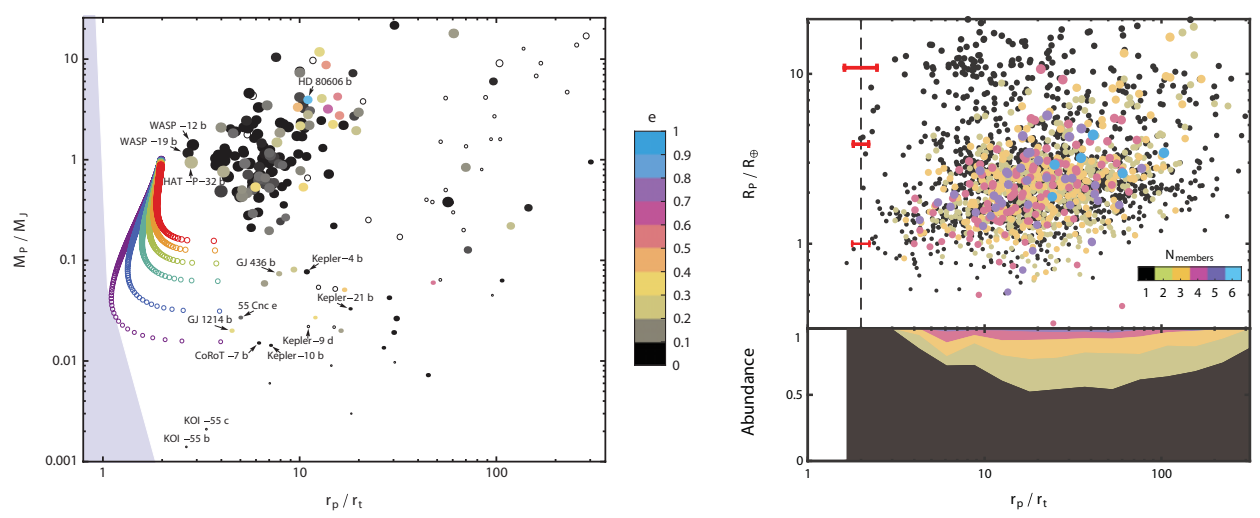

Figure 7. A sample of exoplanets with known masses and radii (left panel). The radius in Earth radii of Kepler planet candidates versus pericenter distance (right panel).

the sample is highly biased against low-mass planets, simply because we need transit surveys to determine the planet's size. In addition, the eccentricities for many low-mass planets are poorly constrained, and as a result, their periastron separations may have been overestimated. This highlights the importance of conducting a survey that is capable of detecting close-in, low-mass planets, such as the Kepler mission.

In the right panel of Fig. 7, we plot the distribution of Kepler candidate radii as a function of their periastron separations (taken to be equal to the semimajor axes) divided by their tidal radii. Unfortunately, we have to use the density of planets in our solar system which have similar sizes to estimate the mass of each candidate. The red bars show the range of $r_{\mathrm{p}} / r_{\mathrm{t}}$ values calculated with two limiting densities at each typical size. The color denotes multiplicity. Intriguingly, the candidates found in multiple systems tend to lie further from their parent stars, and none of the candidates around $2 r_{\mathrm{t}}$ are observed to belong to a multiple candidate system (although they might have distant, unobserved siblings). The paucity of very close-in exoplanet candidates in multiple systems might suggest that the ordered, gentle migration that typifies most of these systems may not be universal, and that some systems may evolve via intense periods of dynamical evolution.

\section{References}

Faber, J. A., Rasio, F. A., \& Willems, B. 2005, Icarus, 175, 248

Fryxell, B., et al. 2000, ApJS, 131, 273

Guillochon, J., Ramirez-Ruiz, E., \& Lin, D. 2011, ApJ, 732, 74

Hjellming, M. S. \& Webbink, R. F. 1987, ApJ, 318, 794

Kozai, Y. 1962, AJ, 67, 591

Lin, D. N. C., Bodenheimer, P., \& Richardson, D. C. 1996, Nature, 380, 606

Lin, D. N. C. \& Ida, S. 1997, ApJ, 477, 781

Nettelmann, N., Holst, B., Kietzmann, A., French, M., Redmer, R., \& Blaschke, D. 2008, ApJ, 683,1217

Ohta, Y., Taruya, A., \& Suto, Y. 2005, ApJ, 622, 1118

Pollack, J. B., Hubickyj, O., Bodenheimer, P., Lissauer, J. J., Podolak, M., \& Greenzweig, Y. 1996, Icarus, 124,62

Rappaport, S., Verbunt, F., \& Joss, P. C. 1983, ApJ, 275, 713

Rasio, F. A. \& Ford, E. B. 1996, Science, 274, 954

Triaud, A. H. M. J., et al. 2010, A\&BA, 524, A25

Winn, J. N., Fabrycky, D., Albrecht, S., \& Johnson, J. A. 2010, ApJ, 718, L145

Wu, Y. \& Lithwick, Y. 2011, ApJ, 735, 109 